\title{
A Study on the Role of Sentence Structure Analysis in English Learning
}

\author{
Xiaoli Yu \\ The English Department, Zhejiang Yuexiu University of Foreign Languages, Shaoxing, China \\ Email:704991373@qq.com
}

How to cite this paper: Yu, X.L. (2021) A Study on the Role of Sentence Structure Analysis in English Learning. Open Access Library Journal, 8: e7859.

https://doi.org/10.4236/oalib.1107859

Received: August 17, 2021

Accepted: September 25, 2021

Published: September 28, 2021

Copyright $\odot 2021$ by author(s) and Open Access Library Inc.

This work is licensed under the Creative Commons Attribution International License (CC BY 4.0).

http://creativecommons.org/licenses/by/4.0/

(c) (i) Open Access

\begin{abstract}
In the introduction part, the paper points out that many students feel that long difficult sentences in English are really very difficult and bring great difficulties in understanding, but the long difficult sentences in English are ubiquitous, which is determined by the language characteristics of English itself. So in the methodology part, this paper first analyzes the structural features of English sentences which are demonstrated mainly in two aspects and introduces the English sentence structure analysis method which explores the different sentence structure types and uses a case study to demonstrate the detailed usage of this method. Then in the discussion part, the paper discusses the role of sentence structure analysis in English listening, reading comprehension, cloze test, translation, oral expression and writing before it finally works out the conclusion that the analysis of English sentence structure plays an universal role in English learning, and students of English major should master this method through certain practice like systematically mastering grammar, doing more analysis in reading and practicing and reading aloud so as to fundamentally improve their English levels.
\end{abstract}

\section{Subject Areas}

Linguistics

\section{Keywords}

Long Difficult Sentences in English, Structural Characteristics, Structural Analysis, Role

\section{Introduction}

Many college students have such a puzzle: the words of a long sentence are basically known, but it is difficult to completely understand its meaning. Such a sen- 
tence will make the student's train of thought blocked, unable to continue to read on. Once the sentence is the exact source of the answer to a question, the student is more likely to mischoose the answer (Tan, 2017: 60) [1]. If such long difficult sentences appear in the listening part, students are even at a loss, not knowing what to start with. But long and difficult English sentences are ubiquitous, so to dig out the reason behind the long and difficult English sentences is necessary. The reason may be that English is a hypostasis language, so its sentence structure is very rigorous and hierarchical. Phrases are linked together by related words that indicate a certain relationship, thus long and complex sentences are formed. In this paper, based on the teaching experience of comprehensive English for English majors, the author cites some typical examples from daily teaching and subject guidance to explore the structural features of English long and difficult sentences and the role of Structural analysis in English listening, speaking, reading, writing and translation.

Let's look at the following sentence:

(1) There was no flinching and no thought of giving in; and by what seemed almost a miracle to those outside these islands, though we ourselves never doubted it, we now find ourselves in a position where I say that we can be sure that we have only to persevere to conquer (He Zhaoxiong, 2013) [2].

In term of punctuation, we know that the above sentence is made up of two sentences of which the second sentence is long and difficult; therefore the two sentences connected by semicolons occupy more than three lines of the textbook, which brings difficulties for students to correctly understand the meaning of it. In fact, this second sentence is a subject-subordinate compound sentence, which consists of a main clause and an adverbial clause. The reason why this sentence is long and difficult is that the adverbial clause contains a parenthesis, and the subject complement in the main clause contains an adverbial clause. It is this kind of sentence packaged in sentence and layer upon layer of English sentence structure that causes the difficulty of the comprehension of English sentences. To overcome such long and difficult English sentences, we are not without any method but the method is quite simple and easy to use, which is the structural analysis method.

\section{Methodology}

\subsection{Literature Review of English Long and Difficult Sentences}

At present, the research on English long and difficult sentences mainly focuses on the translation skills of English long and difficult sentences. For example, Jia Tianhua and Yang Ying (2020) [3], based on the theory of functional equivalence, adopted the method of case analysis to study the sentence patterns and structures of long and difficult sentences in college English, elaborated the linguistic features of Chinese and English, and summarized practical translation methods such as inversion method, translation method, synthesis method, combination method, parenthesis method, increment method and subtraction me- 
thod. Zhou Jin (2018) [4] proposed to take core sentence analysis as the guide to explore how to transform the parts of speech of core components and how to sort out the relationship between core sentences in the translation process of long and difficult sentences so as to improve the accuracy of the translation. Some researches are reports and studies on translation practices in professional areas. For example, the practice report on translation of information technology news texts (Liu Chuankun, 2017) [5], the practice report on solving the problem of English long difficult sentences in the Opening Speech of the Fifth Round of China-US Strategic and Economic Dialogue (Gao Xiaoyan, 2014) [6], the experiment report of Huo Jinchen (2014) [7] on the comprehension strategies of Long difficult sentences in English-Chinese consecutive interpretation, and $\mathrm{Ma}$ Ruimin (2014) [8] Ma, a practical report of E-C translation of long and difficult English sentences in brief introduction type of texts. These research reports all involve translation techniques and strategies of long and difficult sentences, some structural features of English long and difficult sentences, hypotaxis and parataxis between English and Chinese and the differences in word order between English and Chinese, etc. However, there are few studies on the structural features and processing analysis of English long and difficult sentences, as well as on other English fields other than reading, such as listening, speaking, reading and writing. A few researches related to the understanding of English long and difficult sentences are also for the service of translation. For example, a brief discussion on the structural analysis and translation of English long and difficult sentences (Li Yuanhui, 2017) [9] is the one in case. Or a few are about the relationship between English long and difficult sentences and the importance of English grammar. For example, Zhou Jun (2013) [10] viewed the importance of grammar in understanding long and difficult English sentences. Under such a research background, this paper, starting from the structure of English long and difficult sentences, explores the role of structural analysis of English long and difficult sentences in English listening, speaking, reading, writing and translation, in another word, its role in comprehensive English learning.

\subsection{The Structural Features of English Sentences}

The characteristics of English language determine the necessity for us to grasp the characteristics of English sentence structure. English is a hypotactic language. The American Heritage Dictionary defines hypotaxis as "the dependent or subordinate construction or relationship of clauses with connectives". According to Qi Yucun's Encyclopedia of Linguistics, hypohysis refers to the combination of a subordinate language unit with a related word and the main language unit. From the sentence level, we can see that hypotaxis in a broad sense is also proposed by some scholars from the word level. For example, hypotaxis refers to the connection of words or sentences by means of linguistic form (including lexical and morphological means).The categories of English grammar can all be given form in English (Huang Dexian 2004: 19) [11] English sentences pay attention to hypotaxis, that is, words or clauses in a sentence are connected 
by linguistic forms, such as conjunctions and related words to express grammatical meaning and logical relations. Sentence pays attention to form, structure integrity and meaning presentation by form (Wu Jingni 2000: 25) [12] which determines some notable features of English sentence structure.

\subsubsection{Subordination}

The subordinate structure is one of the most important features of modern English. By means of certain relative words and connecting devices, clauses or phrases can act as the main component or subordinate component of the whole sentence, making the sentence contained within the sentence, interlocking with each other thus showing long and complex characteristics. Sometimes, a sentence may cover an entire paragraph. Take the following sentence as an example:

(2) Alan Austen, as nervous as a kitten, went up certain dark and creaky stairs in the neighborhood of Pell Street, and peered about for a long time on the dim hallway before he found the name he wanted written obscurely on one of the doors (He zhaoxiong, 2013) [2].

The main body of the sentence is "Alan Austen went up stairs and peered about for a long time" while in the middle the sentence "as nervous as a kitten" is a comparative adverbial clause which separates the subject from the predicate part "in the neighborhood of Pell Street" and "on the dim hallway" in the later part are both prepositional phrases served as adverbials of place while the preposition "before" is used to connect the main clause with an adverbial clause of time. And the following subordinate clause contains another attributive clause"he wanted", of which "that" is omitted with "written obscurely on one of the doors" as the object complement, and this complement again is composed of an adverb "obscurely" and a phrasal preposition "on one of the doors" which are used as adverbials of manner and place respectively. Through such analysis, we can see that the main part of the sentence is actually very simple, which is composed of a subject and two parallel predicates, i.e. "Alan Austen went up certain dark and creaky stairs and peered about for a long time". The length of the sentence and the complexity of the sentence structure lie in the complexity and verbosity of the subordinate structure in the sentence. As long as we separate the analysis processes of the main clause and subordinate clause, combined with the relationship between them, we can naturally make clear the structure of the whole sentence and hence its meaning.

\subsubsection{End Extension}

According to what Mr. Liu Biqing said in Chinese-English Contrastive Research and Translation, English sentences present an closed form at the beginning and an open one in the end, and attributive modifiers can be post fixed while related prepositions can be connected with modifiers, and sentences can be continuously extended to the end of the sentence (Wu Jingni 2000: 45) [12]. Take the following sentence as an example:

(3) However, they ask for a small room with plain walls where they cannot be 
seen except by the members of their own party, where jackets can come off and they can proceed with the serious business which brought them there (He Zhaoxiong, 2013) [2].

The main body of the sentence is "they ask for a small room". The following prepositional phrase "with plain walls" is a postponed attributive, modifying the current word before it "room". And behind it the two adverbial clauses introduced by "where" are also used to modify the antecedent "room". In the second adverbial clause, two other clauses are joined by "and" of which the second clause once again contains an attributive clause introduced by "which", used to modify the preceding "the serious business". Through this analysis, we find that the central gist of the sentence is expressed first and placed at the beginning of the sentence, followed by the modifying part which is connected by means such as relative words, prepositions and phrases, and thus the sentence is greatly extended, showing the end-of-sentence extension characteristics of English sentences.

\subsection{English Sentence Structure Analysis Method}

\subsubsection{Sentence Structure Type}

It is the characteristics of English sentence structures that determine the complexity of English sentences, so sentence structure analysis is particularly important in sentence understanding and sentence application, for which we introduce sentence structure analysis method. In order to flexibly apply this method, we should first make clear the various components of a sentence, namely sentence components, which mainly include subject, predicate, object, attributive adverbial, complement and appositive. According to the sentence structure, English sentences can be divided into three types: simple sentence, compound sentence and complex sentence. Compound sentences and complex sentences are just obtained by connecting simple sentences with some connecting means, such as parallel conjunctions or subordinate conjunctions. Therefore, after mastering the basic sentence patterns of simple sentences, complex sentences can be analyzed by handling the connecting parts and simple sentences are made up of five basic sentence patterns, which are as follows: I. Subject + linking verb + predicate: She is a teacher. II. Subject + intransitive verb (phrase) (adverbial): It rises very quickly. III. Subject + transitive verb (phrase) + object: The widespread use of the automobile changed our society and culture. / The whole family depends on him to earn money. Here we should pay attention to the use of an infinitive or gerund phrase as an object in this sentence pattern. IV. Subject + transitive verb + indirect object + direct object: He brings me his favorite book. There are other verbs for this type: give, pass, tell, show, leave, offer, promise, teach, pass, etc. V. Subject + object + object complement: He made her happy. / My mother encouraged me to keep on. / All the students consider him a good teacher. The above are adjectives, infinitives and noun phrases used as complements. The subject, object and predicate in the above five sentence patterns can be expanded into corresponding clauses to become compound sentences. In addition, it 
should be noted that which type of sentence pattern the sentence adopts depends entirely on the usage of the predicate verb, so paying attention to the form of the verb in the sentence can help us identify the sentence pattern and grasp the sentence structure.

\subsubsection{Case Study}

Through such classification, we can classify a sentence into a certain category no matter how long it is, and quickly clarify the frame structure of the sentence by analyzing each component of the sentence. When it comes to complex sentences, we can make a layer-by-layer analysis, and then the meaning of the difficult sentences will be clear. Now let's look at the sentence at the beginning of the passage again.

(1) There was no flinching and no thought of giving in; and by what seemed almost a miracle to those outside these islands, though we ourselves never doubted it, we now find ourselves in a position where I say that we can be sure that we have only to persevere to conquer (He Zhaoxiong, 2013) [13].

The sentence before the first semicolon is separated from the sentence after it by "and", forming a parallel structure. Let's analyze the first clause of which the subject is "There", so the whole sentence is of a "There be" construction which play the role of subject and link verb respectively. Therefore, we can determine the sentence a subject-link verb-predicative structure. The predicate is two noun phrases joined by "and" of which the central words are respectively two nouns"flinching" and "thought". And the "no" before each noun is a negative adjective determiner while the phrasal preposition "of giving in" after the "thought" is a postponed attributive, modifying the antecedent "thought" before it. Through such an analysis, the structure of the whole sentence and the specific components of each part are quite clear.

Now let's take a look at the later clause, which is more complicated, is exactly the difficulty of the whole sentence. This is a complex sentence and let's find its main clause first-we now find ourselves in a position-after which the sentence "where I say that we can be sure that we have only to persevere to conquer" is an adverbial led by "where" and "where" equals to "in which". In this adverbial clause there is another object clause led by "that" and the object clause contains another object clause led by "that". It is this layered, overlapping structure that makes English sentences long and complex. Now look at the part before the main sentence. It's actually a phrasal preposition led by "by" and its object is a noun phrase led by "what". In this noun phrase, the infinitive phrase after "miracle" is an adverbial meaning "for those outside our island nations", after which the subordinate clause led by "though" is a parenthetical part of which the "it" refers to the "miracle" before it.

Through the above analysis, we grasp the whole frame of the sentence, and the meaning is clear at a glance. Look at the following translation:

没有退缩、屈服的想法。在我们岛国之外的人看来, 这几乎是一个奇迹。 尽管我们自己从来没有怀疑过这个奇迹, 而通过创造这样的奇迹, 我们现在 


\section{Discussion}

\subsection{The Role of Sentence Structure Analysis}

The importance of mastering English sentence structure analysis is inestimable. It has extraordinary performance in all aspects of English study, including English listening, speaking, reading, writing and translating.

\subsubsection{The Role of Sentence Structure Analysis in English Listening}

Let's first look at the role of sentence structure analysis in English listening. Having a good grasp of the structure of English sentences can help us have a good prediction of English sentences in the process of listening which enables us to predict the likelihood of the next part as to what elements and what structures are to come, thus we can effectively improve our listening efficiency. Take the following sentence from passage dictation as an example:

(4) Consumer behavior essentially refers to how and why people make the purchase decisions they do (Essay dictation passage 42).

The students find it difficult to dictate this sentence because the sentence is long and the structure is complex, so it is difficult to write down the whole sentence within a short time. In particular, some students are not sure about the structure of the sentence. They just write down every word they hear, so that the sentence becomes a long sentence with 15 words with its meaning being cut, logic being lost, so the sentence is naturally long and difficult. The correct way to operate is to grasp it as a whole which means that, before listening, we should have a psychological preparation, ready to grasp the main part of the sentence, so that we have a prediction of each part of the sentence. When we hear "consumer behavior essentially", we expect it to be followed by a predicate verb or verb phrase, which is sure enough, there it comes "refers to". When we hear "how and why", we can predict that it is going to be a noun clause led by "how and why" acting as the object part. Under the operation of such a predictive mechanism, we will find that English listening becomes simpler and the accuracy is improved accordingly. The mastery of sentence structure and composition in the listening process allows us to divide a long and difficult sentence into several meaningful blocks according to the sentence structure. In this way, a sentence becomes several constituent parts, which instantly becomes easier to remember and easier to write down. Look at this sentence again. We find that "consumer behavior" is the subject of the sentence with "refers to" as the predicate and in front of it the word" essentially" is the adverbial modifying the predicate verb. So by the sentence structure, we can anticipate a verb or a phrasal verb that is to come when we hear the word "essentially". In the following object clause, we predict that it should be the predicate part of the object clause while "the purchase" should be the objective case of the verb "make" when we hear "make the purchase", but we soon hear the "decisions" that follow. That's when we should 
react quickly that "purchase" Should be a nominal determiner which is used to modify the central word "decisions" in the objective part. And in the end of the sentence, "they do" is an attributive clause with "that" omitted that is used to modify the antecedent "the purchase decisions". In this way, the grasp of the sentence structure and its compositions in our listening process has played a big role. But there is a certain distance between mastering this method and applying it flexibly in listening practice, which requires us to practice and consolidate it consciously in daily learning for a long time, so that it can form a kind of conditioned reflex and instinct. Because listening itself is time-bound and uncontrollable, it will be a challenge rather than a help to try to use this skill without your proficient mastering of it.

\subsubsection{The Role of Sentence Structure Analysis in English Reading}

Grasping the structure of English sentences helps us to improve the speed and accuracy of English reading. The investigation of English long difficult sentences which are complex in structure is very common, especially in the reading comprehension part of English level or professional examination. But when we use English sentence structure analysis, the problem can be easily solved. Take the following example:

(5) Which of the following statements about Sparrow's research is CORRECT?

[A] We remember people and things as much as before.

[B] We remember more Internet connections than before.

[C] We pay equal attention to location and content of information.

[D] We tend to remember location rather than the core of facts.

(TEST FOR ENGLISH MAJORS GRADE FOUR 2015) [14]

According to the question, we look for the key words and spot "Sparrow's research", and go to the second paragraph of the passage:

Research conducted by Betsy Sparrow, an assistant professor of psychology at Columbia University, has identified three new realities about how we process information in the Internet age. First, her experiments showed that when we don't know the answer to a question, we now think about where we can find the nearest Web connection instead of the subject of the question itself. A second revelation is that when we expect to be able to find information again later on, we don't remember it as well as when we think it might become unavailable. And then there is the researchers' final observation: the expectation that we'll be able to locate information down the line leads us to form a memory not of the fact itself but of where we'll be able to find it.

(TEST FOR ENGLISH MAJORS GRADE FOUR 2015) [14]

In the process of reading, we should eliminate the irrelevant information and grasp the key information, so as to improve the speed of reading. In this section, "an assistant professor of psychology at Columbia University" is the appositive of "Betsy Sparrow", which we can tell at a glance, and when we read, we can just ignore it and skim to the predicates below "has identified" and the object part "three new realities about how we process information in the Internet age". In 
the object part "three new realities" is the key. The question is about which information is correct about "Sparrow's research". So it's important to look at the three new facts about the study. With the help of some clue words "First", "A second revelation", and "the researchers' final observation" combined with the options, we quickly locate the last fact: the expectation that we'll be able to locate information down the line leads us to form a memory not of the fact itself but of where we'll be able to find it. Analyzing this sentence, we find that the subject is "the expectation", the predicate is "leads", the object is "us", and the phrasal infinitive after it is the object complement. This object complement is a phrasal verb. A prepositional phrase is used as a postpositive attributive to modify the antecedent "a memory", which is also the object of the verb phrase. And the object part of this prepositional phrase is made up of two parallel structures. The first is a noun phrase, while the second is more complex, which is composed of a nominal clause which is led by "where" and "where" equates "the place in which". Analyzed in this way, the meaning of the sentence is worked out which fits the choice $[D]$. We tend to remember location rather than the core of facts, so the answer is D.

In this process, because we grasp the structure of English sentences, so we do not need to read word by word, subjectively and actively omitting some irrelevant information, and at the same time, we quickly grasp the key information, and through the judgment of sentence structure components, we quickly work out the answer. This is the application of sentence structure analysis in reading.

\subsubsection{The role of Sentence Structure Analysis in Cloze}

English sentence structure analysis is equally useful, or even better, when dealing with cloze questions in English tests. The students' answers to cloze questions are not very ideal. They generally find it difficult and can't keep up with the speed. This is related to our English foundation, reading ability and reading speed. However, if students have mastered the method of syntactic structure analysis, they will get twice the result with half the effort.

You don't need to read the whole text to answer this type of question. In order to save time and speed up, we usually have a quick glance at the first sentence of the whole text, because it often relates to the theme or central idea of the chapter, and then we work directly on the blank in the sentence. By analyzing the structure of the sentence and determining the part of speech and the word composition to be filled in the blanks, we can quickly work out the answer from the choices. Let's look at the following example:

(6) About 2 million jobless Americans fear they'll lose their extended unemployment benefits, which are slated to end next month unless Congress votes to renew them. Their concerns make a new finding all the more: many people eligible for unemployment don't even bother to collect it.

(TEST FOR ENGLISH MAJORS GRADE FOUR 2015) [14]

Because this is the first blank of the cloze test, and the sentence which involves the blank provides not enough information for us to get the correct answer to 
the blank, because it involves the logical relationship between the sentences before and after it. In this case, it is necessary to start with the first sentence from which we can get a quick idea of the topic of the whole passage. This is a complex sentence and the preceding sentence is the main sentence which is talking about that two million unemployed people in the United States fear losing their long-term unemployment benefits. The main clause is followed by an attributive clause led by "which", modifying the antecedent "extended unemployment benefits". It says, the long-term unemployment benefits are scheduled to end next month unless the Congress votes to renew them. Now that we know the first sentence, let's look at the second sentence, which is where the blank is. We can analyze the structure of the sentence to know the part of speech of the word(s) in the blank. The subject of this sentence is "Their concerns", the predicate verb is a causative verb "make", the object is "a new finding". According to the collocation of the verb "make" and combining the qualifying section "all the more" before the blank, we conclude that the word in the blank part should be an adjective because we have the structure of "make something + adjective". This adjective is the complement of the object. Once the part of speech has been determined, let's look at its meaning. At this time we also look at the part after the colon, and according to the function of the colon, we know that it is the explanation and supplement of the previous part. What this sentence says is that many people who are eligible for the benefit are actually unwilling to do so. Through this analysis, we can see that the contents of the two sentences are contradictory to each other, so we filter out "puzzling" from the options which should fit the blank and should be the correct answer.

Cloze test is a comprehensive test of our understanding of English words, phrases, sentences, logic, comprehension and cultural background, etc. However, mastering the English sentence structure analysis method can help us find a shortcut in this comprehensive test and improve our reading speed and the accuracy of our answers to the questions.

\subsubsection{The Role of Sentence Structure Analysis in Sentence Translation}

When we deal with English sentence translation, the English sentence structure analysis method can also help us achieve an efficient, reasonable and correct translation effect. Whether it is from Chinese to English or from English to Chinese, once the basic frame of the sentence is determined through sentence structure analysis, the translation can be done quickly. By analyzing the relationship of each part, the use of specific words, collocation and tenses, we can ensure that the translation is basically reasonable and correct. Let's look at the following sentence:

(7) 我安排他们在小酒吧见面, 但那个小伙子一直都没有来。(turn up) ( $\mathrm{He}$ Zhaoxiong, 2013) [13]

The sentence structure is composed of two clauses, and the relationship between the two clauses is a transitional relationship, so it is a parallel structure connected by "but". Let's translate the first sentence first. Analyzing the struc- 
ture, we find that the subject is “我”, the predicate is a phrasal verb “安排......见 面”, and the object is “他们” while “在小酒吧” is the adverbial of the place where they met which we can deal with by a prepositional phrase and we can use phrasal verb “arrange for somebody to do something” to deal with “安排...... 见 面”. In term of tense, it should be the past perfect tense since the young man didn't come, but I arranged for them to meet at the pub before that. After analysis, this clause can be translated into "I had arranged for them to meet each other at the pub”. Now let's look at the later clause in which the subject is “小伙子”, “没有来” is the predicate part, “那个” is a qualifier and“一直” is an adverb of time, used to modify the predicate verb. The translation can be: “The young man never turned up." The predicate verb should be the phrasal verb given in the bracket and besides, we should pay attention to this the time adverb “一直” is an adverb of time in the past time, so we'd better choose "never" to translate it but not "all the time" in the present tense. Then the translation of the whole sentence comes out as follows:

I had arranged for them to meet each other, but the man never turned up.

There's no doubt that the sentence becomes much clearer and simpler after the analysis of the sentence structure and the translation becomes quite easy, and still the phenomenon of missing translation can also be avoided. Here is another sentence for "E-C" translation:

(8) But we must learn to be equally good at what is short and sharp and what is long and tough (He Zhaoxiong, 2013) [13].

Analyzing the structure of the sentence, we find the subject is "we", the predicate is "learn to be good at" and "equally" is an adverb, modifying the adjective "good" while the object is two nominal phrases led by "what" which are "what is short and sharp" and "what is long and tough". According to the context and the background of the article, we can translate the sentence into “但我们必须学会 同样善于应付短暂而干脆与漫长而艰难的局面(here the “局面” has the meaning of "battle")".

\subsubsection{The Role of Sentence Structure Analysis in Oral English}

The fluency and accuracy of oral English expression not only depends on the vocabulary, expression habits, culture, psychological factors and other elements, but more importantly depends on our ability to choose words and construct sentences, namely the grasp of the English sentence structure. When we are at the primary stage of grasping the structure of English sentences, we can express our thoughts and standardize our language through the concept of sentence structure. Take the following sentence as an example:

（9）因为无法找到一支英国生产的圆珠笔, 我买了一支法国生产的。这支 笔很贵，虽然它极其简单。(He Zhaoxiong, 2013) [13]

To express such a meaning, we start from the structure of the sentence. English sentences are usually expressed in long and difficult sentences such as compound sentences or subjective and subordinate complex sentences while Chinese sentences tend to be expressed in multiple short sentences, so we have to switch 
between the two, and the effective way is through correlatives. Analyzing this sentence, we find that the relationship between the first two clauses is a causal relationship while the relationship between the last two clauses is a transitional relationship, and the last two sentences and the first two sentences can be connected by the relative words of the non-restrictive attributive clause. By doing this analysis, we can quickly form a translation like this in our mind: as I could not find a British-made ballpoint pen, I bought a French one, which was expensive although it was an extremely simple pen.

When our syntactic analysis ability reaches a certain degree of proficiency, our expression will become natural and fast and even form a certain conditioned reflex by which the thoughts we want to express will be classified and arranged almost synchronously according to the syntactic structure of English, so as to form certain English sentences through which we can express our thoughts, convey our emotions, and achieve effective communication. In a word, the role of sentence structure analysis in oral English is obvious, and the cultivation of the ability of sentence structure analysis is an effective way to improve our oral English ability.

\subsubsection{The Role of Sentence Structure Analysis in English Writing}

The key factors that determine the quality of a piece of writing are content relevance, content adequacy, article structure and language quality of which language quality is largely determined by language accuracy and richness of language structure, which inevitably involves the use of sentence structure analysis. Only when we master this method can we make sentences that are grammatically and semantically accurate and reasonable and at the same time, we can try to use various sentence structures to express semantics through sentence structure analysis so as to meet the requirements of accurate sentences and rich sentence patterns as well, which, to some extent, guarantees the language quality of writing and thus realizing the effective improvement of writing level.

\section{Conclusion}

To sum up, we can see that the method of analyzing English sentence structure is of great help in dealing with English sentence comprehension, English reading, English listening, English cloze, English translation, oral English expression and English writing, and it is a very effective method. In a word, English sentence structure analysis is a universally applicable method in the field of English learning. Students of English major should master it to make English learning more effective.

\subsection{Learn Grammar and Go beyond Grammar}

It is very important to master some grammar knowledge in the analysis of sentence structure. If we don't have any grammatical concepts about sentence patterns, part of speech, and subject-verb agreement and so on, and can't apply them flexibly in practice, the analysis of English sentence structure will just be- 
come an empty talk. Therefore, we need to master certain grammar knowledge through systematic learning and practice sentence structure analysis method through grammatical means in the practice of English learning. Then, by a certain time, our grammar knowledge will be internalized into our understanding instinct. By that time, we will reach the point where we learn grammar and go beyond it. Our knowledge of grammar is transformed into an invisible instinct, which helps us to learn English with ease and get twice the result with half the effort.

\subsection{Doing More Analysis in Reading and Practicing}

In the process of reading and practicing, we can consciously do some practice of sentence structure analysis, especially when we encounter complex long and difficult sentences. First grasp the big frame of the sentence, quickly grasping the main idea of the sentence, and then consider the details in terms of words and clauses. The more practice we have, the easier it will be for us to master the structure of sentences until this ability becomes instinctive and our reading ability and reading speed will both be greatly improved. The mastery of sentence structure enables us to better understand the reading material, improving our reading efficiency and increasing our interest in reading.

\subsection{Reading Aloud}

By reading aloud, students can use their language sense to better grasp the structure of sentences and the relationship between clauses, which can also better cultivate their language sense. Some complex long and difficult sentences with complicated structures, long and difficult clauses, become the obstacles to reading, and often in reading out loud can the structure, the relationship between the clauses and the core and secondary part of the sentence be naturally highlighted, so the sentence can be solved by reading out loud to some certain extent.

Still, when we come across some difficult and complex sentence patterns, ask a few more "whys". Only in this way can our English sentence structure analysis ability be continuously improved and finally, can we apply this ability to all aspects of English learning, which can fundamentally improve our English level.

\section{Conflicts of Interest}

The author declares no conflicts of interest.

\section{References}

[1] Tan, L. (2017) Use Translation Practice to Help College Students Understand English Long and Difficult Sentences. New Campus (Theory Edition), 11, 60.

[2] He, Z.X. (2013) An Integrated English Course 4, the Second Version. Shanghai Foreign Language Education Press, Shanghai.

[3] Jia, T.H. and Yang, Y. (2020) Translation Strategies of College English Long and Difficult Sentences from the Perspective of Functional Equivalence Theory. Journal 
of Tianjin Vocational and Technical Normal University, 30, 70-74.

[4] Zhou, J. (2018) Translation of English Long and Difficult Sentences into Chinese under the Guidance of Core Sentence Analysis. Peking University, Beijing.

[5] Liu, C.K. (2017) A Practical Report on the Translation of Information Technology News Texts in English. Beijing University of Posts and Telecommunications, Beijing.

[6] Gao, X.Y. (2014) The Practice Report on Solving the Problem of English Long Difficult Sentences in the Opening Speech of the Fifth Round of China-Us Strategic and Economic Dialogue. Liaocheng University, Liaocheng.

[7] Huo, J.S. (2014) An Experimental Report on Comprehension Strategies of Long and Difficult Sentences in English-Chinese Consecutive Interpretation. Guangdong University of Foreign Studies, Guangzhou.

[8] Ma, R.M. (2014) A Practical Report on the E-C Translation of Brief Introduction Texts. Liaocheng University, Liaocheng.

[9] Li, Y.H. (2017) On the Structural Analysis and Translation of English Long and Difficult Sentences. Reading and Writing (Education Journal), p. 14.

[10] Zhou, J. (2013) The Importance of Grammar in Understanding Long and Difficult English Sentences. Journal of Jiamusi Vocational Institute, 30, 232+255.

[11] Huang, D.X. (2004) Form Syntaxis Parataxis Translation Cavity. Journal of Civil Aviation Flight College of China, 1.

[12] Wu, J.N. (2000) Contrast of Sentence Structure between English and Chinese and Translation of Long Sentences. Journal of Hunan College of Commerce, 7, 122-124.

[13] He, Z.X. (2013) An Integrated English Course 3. Shanghai Foreign Language Education Press, Shanghai.

[14] Wang, H.P. (2014) Spark English Majors 4 Explanation + Standard Prediction Test for English Majors (2015)-Grade Four. Jilin Publishing Group Co. Ltd., Changchun. 